\title{
The dynamics of cyclin B1 distribution during meiosis I in mouse oocytes
}

\author{
Petros Marangos and John Carroll \\ Department of Physiology, University College London, Gower Street, London WC1E 6BT, UK \\ Correspondence should be addressed to J Carroll; Email: j.carroll@ucl.ac.uk
}

\begin{abstract}
Cdk1-cyclin B1 kinase activity drives oocytes through meiotic maturation. It is regulated by the phosphorylation status of cdk1 and by its spatial organisation. Here we used a cyclin B1-green fluorescent protein (GFP) fusion protein to examine the dynamics of cdk1-cyclin B1 distribution during meiosis I (MI) in living mouse oocytes. Microinjection of cyclin B1-GFP accelerated germinal vesicle breakdown (GVBD) and, as previously described, overrides cAMP-mediated meiotic arrest. GVBD was pre-empted by a translocation of cyclin B1-GFP from the cytoplasm to the germinal vesicle (GV). After nuclear accumulation, cyclin B1-GFP localised to the chromatin. The localisation of cyclin B1-GFP is governed by nuclear import and export. In GV intact oocytes, cyclin export was demonstrated by showing that cyclin B1-GFP injected into the GV is exported to the cytoplasm while a similar size dextran is retained. Import was revealed by the finding that cyclin B1-GFP accumulated in the GV when export was inhibited using leptomycin B. These studies show that GVBD in mouse oocytes is sensitive to cyclin B1 abundance and that the changes in distribution of cyclin B1 contribute to progression through MI.
\end{abstract}

Reproduction (2004) 128 153-162

\section{Introduction}

In mammalian oocytes, the cell cycle is arrested in prophase of the first meiotic division. Resumption of the cell cycle is stimulated by a hormonal trigger, or release of the oocyte from the ovarian follicle. These triggers lead to an increase in the activity of maturation promoting factor (MPF), a heterodimer consisting of a kinase, cdk1 and its regulatory partner, cyclin B (cdk1-cyclin B) (Draetta et al. 1989, Hunt 1989, Labbé et al. 1989, Gautier et al. 1990). The increase in MPF activity leads to germinal vesicle breakdown (GVBD) and entry into the first meiotic metaphase (M-phase) (Hashimoto \& Kishimoto 1988, Choi et al. 1991). After a protracted first meiotic M-phase, a decline in MPF activity results in the first meiotic division (Ledan et al. 2001). MPF activity then returns and drives the oocyte into metaphase of meiosis II (MII) where, under the influence of the mos/MAP kinase pathway (Tunquist \& Maller 2003), the cell cycle arrests until fertilisation (Hashimoto \& Kishimoto 1988, Verlhac et al. 1993, Hampl \& Eppig 1995).

Cyclin abundance is an important requirement for activation of cdk1 (Murray \& Kirschner 1989). In mitotic cell cycles, new cyclin synthesis precedes each mitosis (Evans et al. 1983) and exit from mitosis is driven by a ubiquitindependent, proteasome-mediated destruction of cyclin B1 (Glotzer et al. 1991). In mouse oocytes arrested at the germinal vesicle (GV) stage, protein synthesis is not required for the activation of MPF or GVBD (Clarke \& Masui 1983, Hashimoto \& Kishimoto 1988, Downs 1990). In the absence of a requirement for new cyclin B synthesis it is thought that dephosphorylation of pre-MPF laid down during oogenesis is sufficient for MPF activation (Choi et al. 1991, Chesnel \& Eppig 1995, Mitra \& Schultz 1996). Protein synthesis is required to progress through meiosis I (MI), since protein synthesis inhibitors prevent normal MI spindle formation (Clarke \& Masui 1983, Hashimoto \& Kishimoto 1988, Hampl \& Eppig 1995, Winston 1997). It is likely that cyclin B is one of the required proteins since the duration of $\mathrm{MI}$ and increase in MPF activity between GVBD and $\mathrm{MI}$ is correlated with the rate of cyclin synthesis (Hampl \& Eppig 1995, Polanski et al. 1998). Furthermore, injection of cyclin B1 mRNA accelerates or delays $\mathrm{MI}$ depending on the length of the poly-A tail, a mechanism that regulates translational efficiency (Tay et al. 2000, Ledan et al. 2001). These studies suggest that the levels of cyclin B1 play an important role in determining the timing of events in $\mathrm{Ml}$, although the effects of cyclin B1 prior to GVBD are largely unexplored.

An additional aspect important for cdk1-cyclin B activity is its spatial distribution (Pines 1999, Takizawa \& Morgan 2000). Cdk1-cyclin B is cytoplasmic during interphase and enters the nucleus late in prophase prior to nuclear envelope breakdown (NEBD) (Pines \& Hunter 
1991, Ookata et al. 1992, Hagting et al. 1998, Casas et al. 1999). In interphase, cdk1-cyclin B is retained in the cytoplasm in a chromosomal region maintenance protein 1 (CRM1)-dependent manner due to a CRM1-binding nuclear export sequence (Hagting et al. 1998, Toyoshima et al. 1998, Yang et al. 1998) in the cytoplasmic retention signal (Pines \& Hunter 1994). In prophase, nuclear accumulation is driven by a phosphorylation-dependent decrease in CRM1-dependent export (Hagting et al. 1998, Yang et al. 1998) and increase in cyclin import (Hagting et al. 1999, Yang et al. 2001). After NEBD, cdk1-cyclin B associates with the mitotic apparatus, including the spindle poles, the microtubules and chromatin. These studies provide a concise account of the temporal and spatial aspects of cell cycle control during mitosis. To date, the spatial organisation of cdk1-cyclin B in meiosis in mammals has not been investigated.

In this study we have utilised a human cyclin B1-green fluorescent protein (GFP) fusion protein (Hagting et al. 1998, Clute \& Pines 1999) to examine the effects of cyclin B1 abundance on GVBD, the dynamics of cyclin B1 distribution in relation to GVBD and the mechanisms underlying the localisation of cyclin B1 during $\mathrm{MI}$ in mouse oocytes.

\section{Materials and Methods \\ Oocyte collection and culture}

Immature GV stage oocytes were collected from 4- to 6-week-old MF1 mice that had been injected $48 \mathrm{~h}$ previously with $7.5 \mathrm{IU}$ pregnant mare's serum gonadotrophin. Mice were killed by cervical dislocation and the ovaries placed in medium M2 (Fulton \& Whittingham 1978) containing $200 \mu \mathrm{M}$ dibutyryl cyclic adenosine monophosphate (M2 + dbcAMP). Cumulus-enclosed oocytes were recovered from the ovaries by puncturing the surface of the ovary with a 27 guage needle. The released oocytes were collected and washed three times in $\mathrm{M} 2+$ dbcAMP.

\section{Microinjection}

Oocytes were microinjected using a micropipette and Narishige manipulators mounted on a Leica or Nikon inverted microscope. Oocytes were placed in a drop of $\mathrm{M} 2+$ dbcAMP under paraffin oil in a lid of a $3 \mathrm{~cm}$ Falcon culture dish. Cyclin was diluted in injection buffer (120 mM KCl, $20 \mathrm{mM}$ HEPES, pH 7.4). The oocyte was immobilised using a holding pipette and the injection pipette was pushed through the zona pellucida and against the plasma membrane. The pipette was inserted using a brief overcompensation of negative capacitance. Pressure was applied via a pico-pump attached to a nitrogen cylinder. The amount of solution injected was calculated using an eye-piece graticule to obtain an estimate of the diameter of the injection bolus. Oocyte volume was estimated to be $250 \mathrm{pl}$.

\section{Confocal and fluorescence microscopy}

Confocal microscopy was performed using a BioRad micro radiance confocal scan head mounted on a Zeiss axiovert 100TV. Oocytes were placed in a heated chamber (35$37^{\circ} \mathrm{C}$ ) and cyclin B1-GFP was excited using the $488 \mathrm{~nm}$ line of an argon laser. Fluorescence was collected through a 20 or $40 \times 0.75 \mathrm{NA}$ objective. Laser power was set to 1 or $3 \%$ of maximum and images were collected at intervals of 5 or $10 \mathrm{~min}$. Each image was averaged two to three times. For conventional imaging, oocytes were placed in a heated chamber as described above on the stage of a Zeiss Axiovert 100TV. Excitation of cyclin B1-GFP $(490 \mathrm{~nm})$, GFP itself $(490 \mathrm{~nm})$ and rhodamine dextran $(550 \mathrm{~nm})$ was via a monochromater controlled by the Metafluor software (Universal Imaging). Fluorescence was collected through a 20 or $40 \times 0.75$ NA objective. Images were acquired using a Princeton Instruments MicroMax interline cooled CCD camera (Roper Scientific, Buckinghamshire, UK). Camera exposure times varied between 50 and $100 \mathrm{~ms}$. Data were collected and analysed using Universal Imaging Metafluor and Metamorph software.

\section{Kinase assays}

MPF and MAP kinase activities were measured using histone $\mathrm{H} 1$ and myelin basic protein (MBP) kinase assays respectively. The MBP assay has been shown previously to correlate with MAP kinase activity in mouse oocytes as determined by more specific gel or immunoprecipitationbased assays (Verlhac et al. 1993). The protocol was similar to that described elsewhere (Kubiak et al. 1993, Moos et al. 1995). Five eggs (unless stated otherwise) in $2 \mu \mathrm{l}$ HEPES-buffered KSOM were transferred in $3 \mu \mathrm{l}$ storing solution $(10 \mu \mathrm{g} / \mathrm{ml}$ leupeptin, $10 \mu \mathrm{g} / \mathrm{ml}$ aprotinin, $10 \mathrm{mM}$ p-nitrophenyl phosphate, $20 \mathrm{mM} \beta$-glycerophosphate, $0.1 \mathrm{mM}$ sodium orthovanadate, $5 \mathrm{mM}$ EGTA) and immediately frozen on dry ice. The samples were subjected to three freeze-thaw cycles, diluted twice by the addition of two times concentrated kinase buffer containing $60 \mu \mathrm{g} / \mathrm{ml}$ leupeptin, $60 \mu \mathrm{g} / \mathrm{ml}$ aprotinin, $24 \mathrm{mM}$ p-nitrophenyl phosphate, $90 \mathrm{mM} \beta$-glycerophosphate, $4.6 \mathrm{mM}$ sodium orthovanadate, $24 \mathrm{mM}$ EGTA, $24 \mathrm{mM} \mathrm{MgCl}_{2}, 0.2 \mathrm{mM}$ EDTA, $4 \mathrm{mM} \mathrm{NaF}, 1.6 \mathrm{mM}$ dithiothreitol, $2 \mathrm{mg} / \mathrm{ml}$ polyvinyl alcohol, $40 \mathrm{mM}$ MOPS, $0.6 \mathrm{mM}$ ATP, $2 \mathrm{mg} / \mathrm{ml}$ histone $\mathrm{H} 1$ (HIII-S from calf thymus; Sigma, St Louis, MO, USA), $0.5 \mathrm{mg} / \mathrm{ml}$ MBP (from guinea pig brain; Sigma) and $0.25 \mathrm{mCi} / \mathrm{ml}\left[{ }^{32} \mathrm{P}\right]$ ATP. The samples were then incubated at $30^{\circ} \mathrm{C}$ for $30 \mathrm{~min}$. The reaction was stopped by the addition of two times SDS sample buffer (0.125M Tris$\mathrm{HCl}, 4 \%$ SDS, 20\% glycerol, 10\% mercaptoethanol, $0.002 \%$ bromophenol blue) and boiled for 3-5 min. The samples were then analysed with SDS-PAGE followed by autoradiography. The autoradiographs were imaged using 
the Fuji Bas-1000 phosphorimager system and analysed with TINA 2.0 software.

\section{Results}

\section{Exogenous cyclin B1-GFP accelerates GVBD}

The lack of any requirement for protein synthesis for GVBD suggests that the requirements for cyclin B1 synthesis have been met during oogenesis. To test whether exogenous cyclin B1 influences the timing of GVBD we have microinjected cyclin B1-GFP at a range of concentrations. The amounts of cyclin B1-GFP injected were chosen with respect to the levels previously reported in fully grown mouse oocytes (10 pg) (Kanatsu-Sinohara et al. 2000). After microinjection, oocytes were released from meiotic inhibitors and the timing of GVBD and first polar body extrusion was examined. The four concentrations tested significantly accelerated GVBD (Fig. 1Ai). The acceleration was found to be dosedependent: 50\% GVBD taking 150, 60, 45 and $30 \mathrm{~min}$ for controls $(n=33)$ and cyclin injections of $10 \mathrm{pg}$ $(n=44), 20 \mathrm{pg}(n=35)$ and $40 \mathrm{pg}(n=29)$ respectively
(Fig. 1Ai). Similarly, the maximum level of GVBD for controls, $10 \mathrm{pg}, 20 \mathrm{pg}$ and $40 \mathrm{pg}$ injections occurred after 180, 90, 60 and 45 min respectively (Fig. 1Ai).

We confirmed that cyclin B1-GFP accelerated GVBD via premature activation of MPF (Fig. 1Aii). MPF started to increase within $30 \mathrm{~min}$ of injection of $40 \mathrm{pg}$ cyclin B1-GFP and had reached a maximum 25-fold increase over basal by $60 \mathrm{~min}$ after injection. In control oocytes, MPF activation was markedly slower and more modest with a twofold increase after $60 \mathrm{~min}$ and a fourfold increase after $120 \mathrm{~min}$ (Fig. 1Aiii). The induction of MPF activity and GVBD also caused an accelerated time of activation of MAP kinase (compare Fig. 1Aii and iii). After cyclin injection, MAP kinase increased gradually from $30 \mathrm{~min}$ reaching a tenfold increase by $120 \mathrm{~min}$ after injection (Fig. 1Aii). At this time $(120 \mathrm{~min})$, MAP kinase activity remained at basal levels in the controls. Starting around $120 \mathrm{~min}$, the MAP kinase activity increased eightfold by $180 \mathrm{~min}$ (Fig. 1Aiii). Thus, although cyclin injection accelerated the induction of MAP kinase activity, it had little effect on the extent of the increase in activity.

\section{$\mathbf{A}_{\mathbf{i}}$}

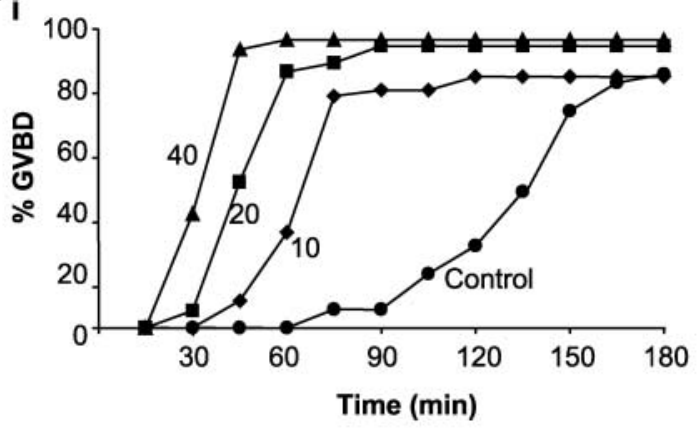

ii

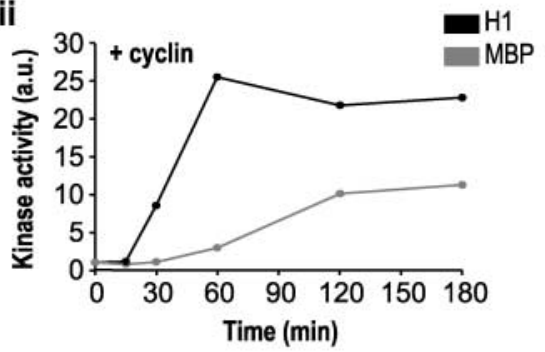

B

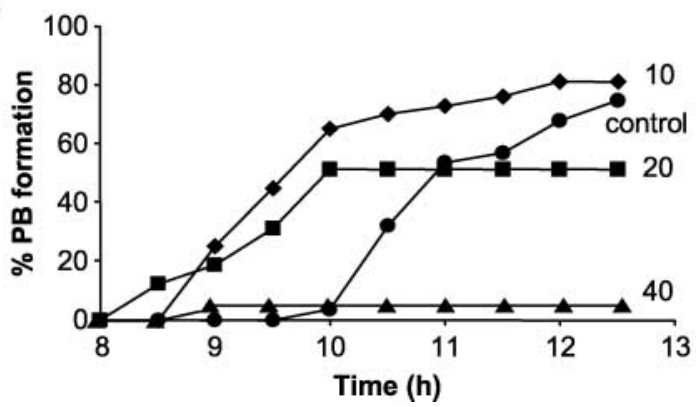

Figure 1 Cyclin B1-GFP accelerates GVBD and MI. (A and B) GV stage oocytes were injected with cyclin B1-GFP in the presence of dbcAMP before culture in dbcAMP-free media. The timing of (A) GVBD and (B) polar body (PB) extrusion, relative to removal from dbcAMP, was monitored using bright field optics. Cyclin B1-GFP accelerated GVBD after microinjection of $10(\bullet), 20(\mathbf{\square})$ or $40 \mathrm{pg}$ (ム) cyclin B1-GFP compared with controls (-) (Ai). H1 kinase and MBP assays for MPF and MAP kinase activities for oocytes injected with 40 pg cyclin B1-GFP (Aii) and control oocytes are presented as the fold increase over basal (Aiii). Note that the kinase activities increase sooner and to a higher level after injection of cyclin B1-GFP. (B) The effect of cyclin B1-GFP on polar body extrusion was shown (a.u., arbitrary units). Lower doses of 10 and 20 pg accelerated polar body extrusion while higher doses increased the proportion of oocytes arrested at MI. Data are pooled from two to three independent experiments with a total of 44,35, 29 and 33 oocytes for the $10 \mathrm{pg}, 20 \mathrm{pg}$, $40 \mathrm{pg}$ and uninjected control groups respectively. 


\section{Cyclin B1-GFP has a concentration-dependent effect on extrusion of the first polar body}

The effects of cyclin B1 on first polar body formation were twofold. First, lower amounts (10 and $20 \mathrm{pg})$ initiated polar body formation earlier $(8.5-9 \mathrm{~h})$ than in control oocytes (10 h) (Fig. 1B). The second effect was that increasing concentrations of cyclin caused a dose-dependent decrease in the ability of oocytes to extrude a polar body (Fig. 1B). These data are consistent with those of Ledan et al. (2001), where over-expression of cyclin B1 caused a similar phenotype. The experiments in the present study extend these observations by determining the effect of a range of concentrations of cyclin B1, rather than relying on different lengths of poly-A tail to provide different amounts of cyclin B1. In oocytes injected with $10 \mathrm{pg}$, similar proportions of oocytes extruded the first polar body compared with controls $(80 \%, 21 / 25$ and $85 \%, 24 / 28$ respectively). After injection of $20 \mathrm{pg}, 50 \%$ $(12 / 24)$ of oocytes extruded a polar body $(P<0.05$ compared with controls) while after injection of $40 \mathrm{pg}$ this was reduced to $5 \%(1 / 19 ; P<0.01$ compared with controls).

\section{Cyclin B1 overrides CAMP-mediated arrest at the GV stage}

Previously, Ledan et al. (2001) showed that overexpression of cyclin B1 was sufficient to override cAMPmediated arrest. Our observation that exogenous cyclin B1 accelerated the rate of GVBD suggested that cyclin B1 abundance is a potent determinant of the timing of entry into MI. To determine if levels of cyclin that accelerate GVBD also override cAMP-mediated arrest we microinjected $40 \mathrm{pg}$ cyclin B1-GFP protein into oocytes maintained in $250 \mu \mathrm{M}$ dbcAMP (Fig. 2A). Cyclin B1-GFP stimulated GVBD at a time similar to the onset of maturation of injected controls cultured in the absence of dbcAMP (Fig. 2A). Fewer oocytes injected and maintained in the presence of dbcAMP underwent GVBD (see Fig. 2A). After $3 \mathrm{~h}, 60 \%$ (41/69) of the cyclin B1-injected oocytes had undergone GVBD in the presence of dbcAMP which was significantly less than the $90 \%$ that had undergone GVBD in its absence $(P<0.01)$. Removal of dbcAMP from the arrested oocytes resulted in GVBD within $60 \mathrm{~min}(87 \%, 21 / 24)$ (Fig. 2B).

\section{GVBD is associated with nuclear localisation of cyclin-GFP}

In mammalian somatic cells, nuclear accumulation of cyclin occurs during prophase just prior to NEBD (Hagting et al. 1998, Pines 1999). Since mammalian oocytes are arrested in a protracted meiotic prophase it was of interest to determine the localisation of cyclin during entry into the first meiotic division. To examine the relationship between cyclin distribution and GVBD, $40 \mathrm{pg}$ cyclin B1-GFP was microinjected into mouse oocytes arrested with dbcAMP. The oocytes were released from dbcAMP and the
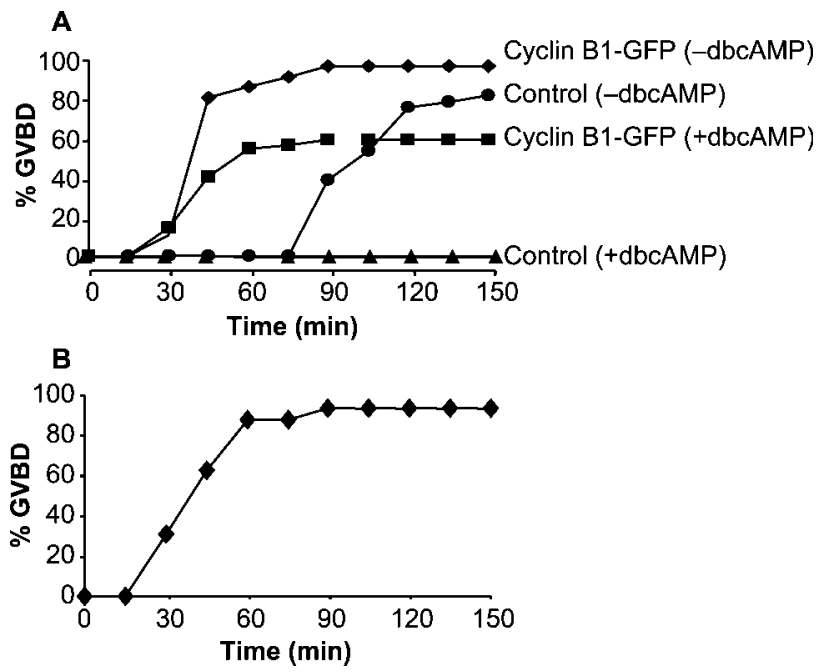

Figure 2 Cyclin B1-GFP overrides CAMP-mediated meiotic arrest. In (A), GV stage oocytes were microinjected with 40pg cyclin B1-GFP in the presence of dbcAMP. The rate of GVBD was then monitored in the continued presence of $\operatorname{dbcAMP}(\boldsymbol{\square})(n=69)$ or after release from dbcAMP $(\bullet)(n=37)$. Control, non-injected oocytes cultured in the presence $(\boldsymbol{\Lambda})(n=43)$ or absence $(\bullet)(n=67)$ of dbcAMP were included for comparison. (B) Cyclin B1-GFP-injected oocytes that failed to undergo GVBD $(n=23)$ in the presence of dbcAMP were released from CAMP and monitored for the presence of a GV. Data are pooled from two to four separate experiments.

localisation of cyclin B1-GFP was monitored using confocal microscopy as the oocytes progressed through GVBD. These experiments revealed that cyclin B1-GFP is initially cytoplasmic with one to two accumulations close to the membrane of the GV (Fig. 3A arrow). These accumulations of cyclin were present in all oocytes and are likely to be the oocyte equivalent of the centrosomes (microtubule organising centres) to which cyclin B1-GFP binds in prophase in somatic cells (Hagting et al. 1998). Cyclin B1-GFP can first be seen entering the nucleus after $20 \mathrm{~min}$ and reached a peak at about $40 \mathrm{~min}$ (Fig. $3 \mathrm{~A}$ and B). GVBD followed peak nuclear accumulation within $10 \mathrm{~min}$. At GVBD, cyclin B1-GFP redistributed in the cytoplasm and some remained associated with the condensed chromatin. Closer examination of the distribution of cyclin within the nucleus suggested that it does not bind to nucleoli and there is no evidence for preferential loading of cyclin-GFP onto the condensing chromatin (see 20 and 40 min timepoints in Fig. 3A). At $40 \mathrm{~min}$, when nuclear cyclin is at a peak, it appeared to be uniformly distributed throughout the nucleus. It is possible that the high level of cyclin-GFP in the nucleus at this stage effectively masked any localisation within the nucleus. The first indication that cyclin B1-GFP localises to the chromatin was after GVBD when the nuclear cyclin B1-GFP redistributes to the cytoplasm. To determine whether cyclin B1-GFP localises to the chromosomes at other stages, MII oocytes were injected with $20 \mathrm{pg}$ cyclin B1-GFP and examined using confocal microscopy. The spindle poles and microtubules and chromatin were decorated with cyclin B1-GFP (Fig. 3C). 
A
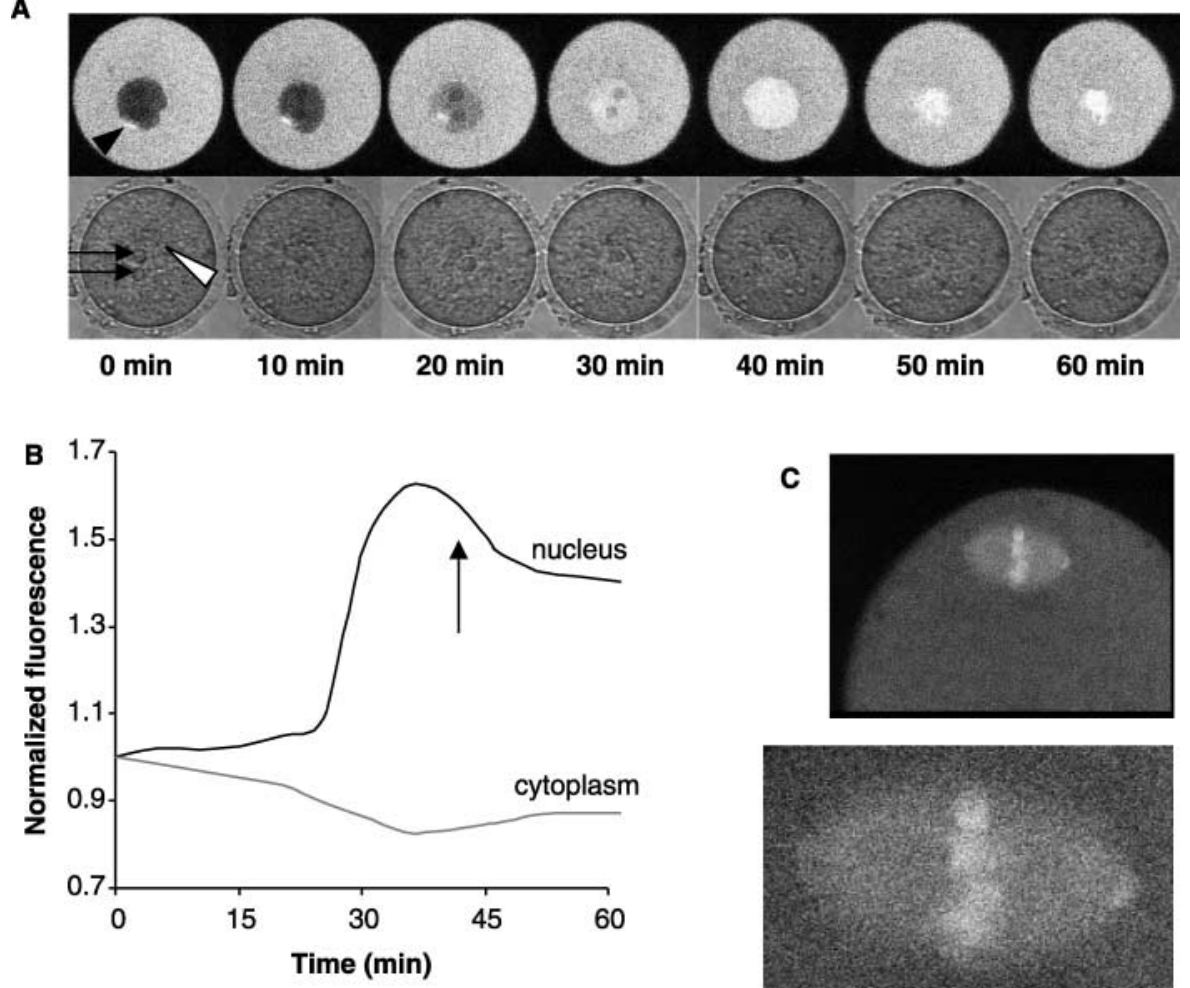

C
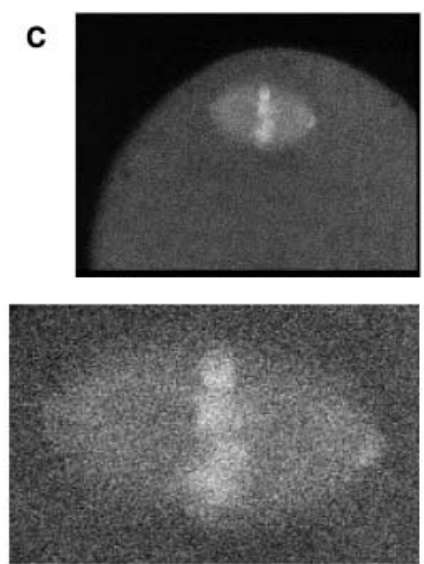

D

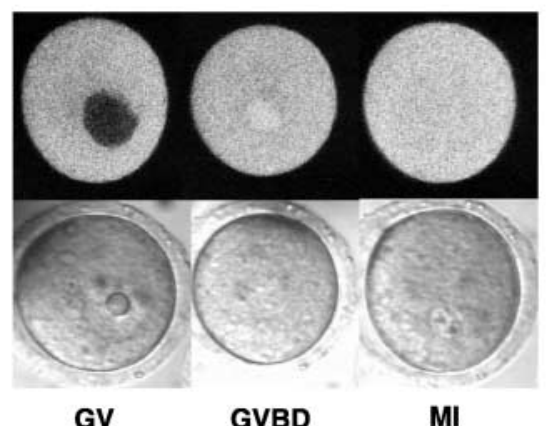

Figure 3 Cyclin B1-GFP enters the GV just prior to GVBD. Oocytes were microinjected with cyclin B1-GFP and confocal microscopy was used to determine its localisation during GVBD. In (A), confocal scans of cyclin B1-GFP recorded every 10 min are shown in the top panel with corresponding bright field images in the lower panel. The black arrow in the first image of the top panel highlights a concentration of cyclin B1-GFP (see Results) that was apparent in all oocytes examined $(n=15)$. The large arrowhead on the first image of the lower panel points to the GV while the thin arrows point to the nucleoli. The cyclin-GFP can be seen to accumulate in the GV then diffuse to the cytoplasm at GVBD that takes place between 40 and $50 \mathrm{~min}$. The last image in the series shows a remnant of cyclin B1-GFP fluorescence on what we assume to be the condensing $\mathrm{Ml}$ chromosomes. (B) A plot of the fluorescence intensities in the nucleus and cytoplasm during the course of the experiment is shown (representative of 12 oocytes examined). The arrow signifies the time at which GVBD was confirmed. In (C), MII stage oocytes were injected with cyclin B1-GFP to determine whether it localised to the metaphase chromosomes. Note that cyclin B1-GFP attaches to the MII spindle including the metaphase plate. In (D), GV stage oocytes were injected with $70 \mathrm{kDa}$ fluorescein dextran and monitored through GVBD with confocal microscopy $(n=8)$. Images are shown when oocytes are at the GV stage, at GVBD and $1 \mathrm{~h}$ after GVBD. Note that fluorescence in the region of the GV was only detected after GVBD.

To confirm that the localisation in the GV was specific to the cyclin B1-GFP, oocytes were injected with 70kDa fluorescein dextran. The localisation of the $70 \mathrm{kDa}$ fluorescein dextran was compared with that of the $97 \mathrm{kDa}$ cyclin-B1GFP fusion protein during GVBD (Fig. 3D). These data showed that the dextran was initially excluded from the nucleus and simply diffuses into the nuclear region after GVBD. There were no perinuclear accumulations of fluorescence or preferential accumulation of fluorescence in the GV prior to GVBD (Fig. 3D).

\section{Cyclin B1 is actively exported from the GV}

Cyclin B1 in somatic cells is retained in the cytoplasm by a nuclear export signal. We examined whether similar mechanisms were controlling cyclin B1 distribution in MI. To visualise a nuclear export mechanism, cyclin B1-GFP 
was microinjected directly into the GV of oocytes maintained in dbcAMP. Imaging experiments revealed that the nuclear cyclin was exported from the GV from the time of the first measurement, $5 \mathrm{~min}$ after the start of recording (Fig. 4A and D). The gradual loss of cyclin supports the idea that it is actively exported rather than being a result of GVBD, where loss from the nucleus would be expected to be more abrupt. In addition, bright field observation of the oocyte after the completion of the experiment revealed that the GV remained intact (not shown).

The cyclin component of the cyclin B1-GFP was necessary for nuclear localisation since GFP alone was not retained within the nucleus. Due to its smaller size, GFP simply diffused throughout the nucleus and cytoplasm within $5 \mathrm{~min}$ of injection (Fig. 4B). As a further test that the decrease in cyclin B1-GFP fluorescence from the nucleus was not due to simple diffusion, $70 \mathrm{kDa}$ fluorescein dextran was injected into the nucleus. The $70 \mathrm{kDa}$ dextran was retained in the nucleus for the duration of the 2-h observation (Fig. 4C). Thus the export from the nucleus is a feature specific to cyclin B1-GFP.

\section{Inhibition of nuclear export accelerates accumulation of nuclear cyclin B1-GFP}

To examine the kinetics and mechanism of cyclin B1-GFP nuclear import during $\mathrm{MI}$, leptomycin $\mathrm{B}$ was used to inhibit CRM1-dependent nuclear export (Fornerod et al. 1997, Hagting et al. 1998, Kudo et al. 1998, Yang et al. 1998) while monitoring nuclear import. When cyclin B1GFP-injected oocytes $(2-3 \mathrm{pg})$ were incubated with $20 \mathrm{nM}$ leptomycin B, nuclear accumulation of the cyclin B1-GFP was apparent from the time of the first recording 5-10 min after injection (Fig. $5 \mathrm{~A}$ and $\mathrm{B}$ ). In contrast to this immediate increase in nuclear cyclin B1-GFP, oocytes undergoing GVBD in the absence of leptomycin B did not show nuclear accumulation in the first $20 \mathrm{~min}$ of the experiment (Fig. 5A and B). This observation was quantified by determining the change in fluorescence intensity in the GV that takes place over a $10 \mathrm{~min}$ interval at the start of the experiment in the presence and absence of leptomycin $\mathrm{B}$ using the formula $\left(\mathrm{Fl}_{10 \min }-\mathrm{Fl}_{0 \text { min }}\right) / \mathrm{Fl}_{0 \text { min; }} ; \mathrm{Fl}$, fluorescence intensity unit. This analysis confirmed that in the first $10 \mathrm{~min}$ of the experiment there was significantly greater nuclear accumulation of cyclin B1-GFP in the presence of leptomycin compared with controls (Fig. 5C). In addition, we measured the change in fluorescence in the GV in control oocytes just prior to GVBD to determine whether inhibition of export is sufficient to explain the rapid accumulation just prior to GVBD. The data showed that the change in fluorescence was greater just prior to GVBD than that seen in the presence of leptomycin B soon after release from dbcAMP (Fig. 5C). This suggested

\section{A Cyclin B1-GFP}

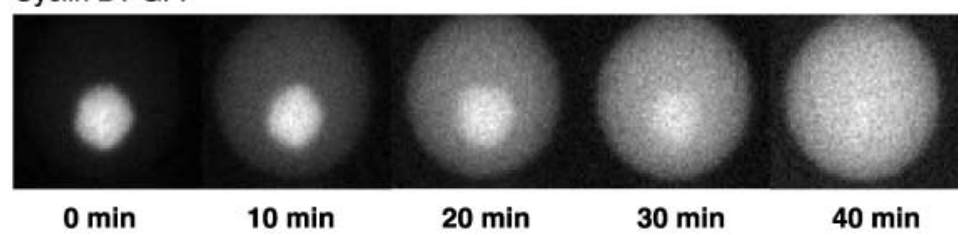

B GFP

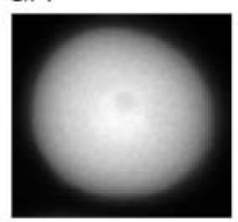

C Fluorescein-dextran

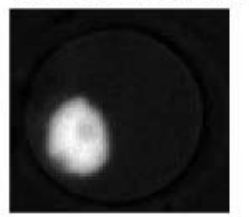

$0 \mathrm{~min}$

D

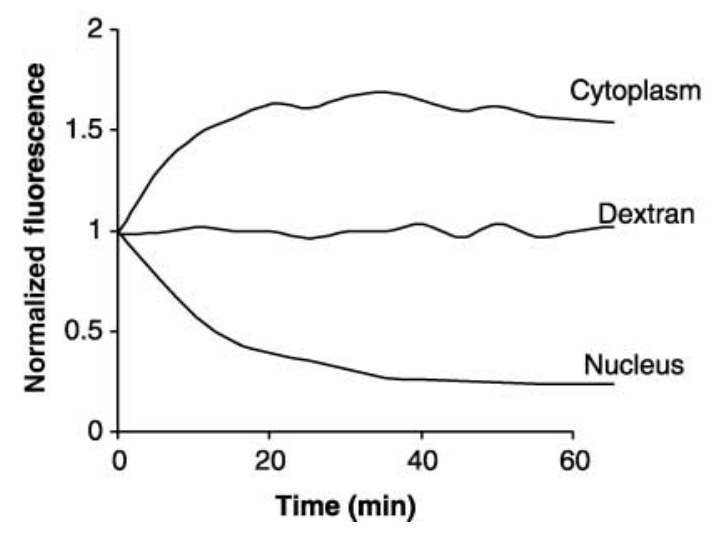

Figure 4 Cyclin B1-GFP is actively exported from the GV. (A) Cyclin B1-GFP was injected into the GV of oocytes maintained in dbcAMP and the redistribution was monitored using confocal microscopy. The export of cyclin B1-GFP is specific because nuclear injection of (B) GFP and (C) $70 \mathrm{kDa}$ fluorescein dextran do not show similar redistribution. (D) A plot of the fluorescence intensities of cyclin B1-GFP in the nucleus and cytoplasm can be compared with the constant level of nuclear fluorescence after injection of $70 \mathrm{kDa}$ fluorescein dextran. GFP redistribution is not plotted on the graph as it had diffused from the GV in the $3-5 \mathrm{~min}$ between injection and imaging. Data presented are representative of eight cyclin B1-GFP-injected oocytes, ten fluorescein dextran-injected oocytes and ten GFPinjected oocytes. 
A

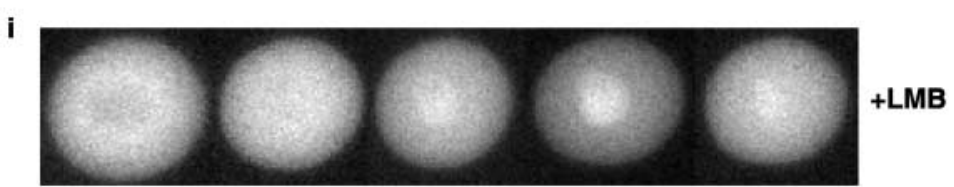

ii

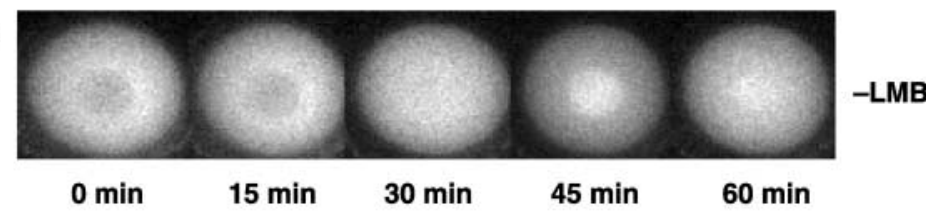

B

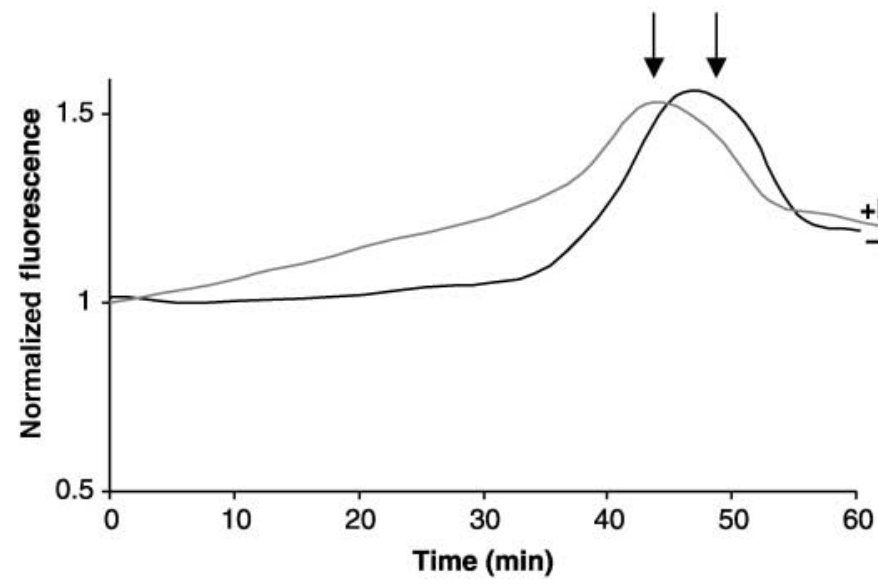

C

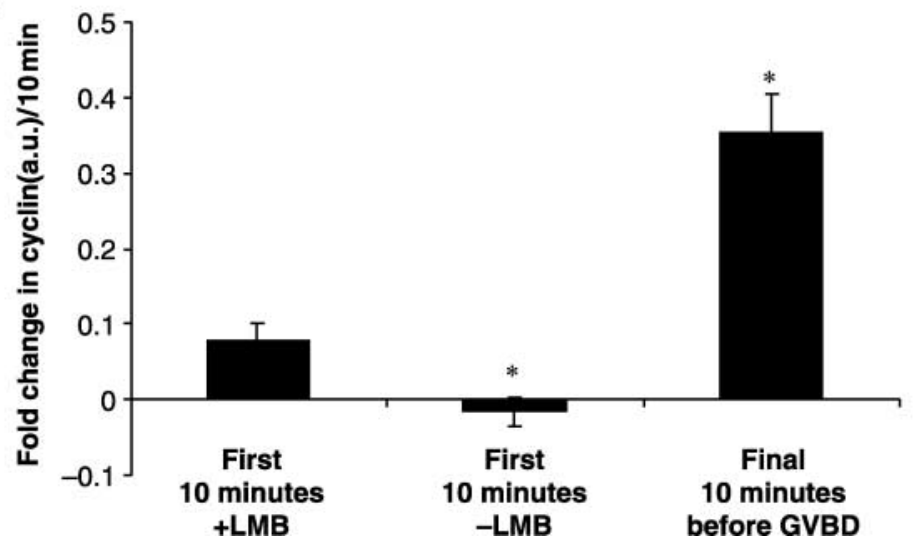

Figure 5 Inhibition of nuclear export leads to nuclear accumulation of cyclin B1-GFP. Oocytes were injected with cyclin B1-GFP (2-3pg) and the timecourse of accumulation in the GV was monitored over $60 \mathrm{~min}$ in the $(\mathrm{Ai})$ presence $(n=12)$ or (Aii) absence $(n=11)$ of leptomycin B (LMB). (B) Fluorescence intensity plots and the representative images (Ai and Aii) show the temporal and spatial redistribution of cyclin B1-GFP. The arrows on (B) show the time at which GVBD was confirmed. Note that in the presence of leptomycin B cyclin B1-GFP accumulates in the GV from the start of the recording. This is quantified in (C) by comparing the relative fluorescence change in the GV in the first $10 \mathrm{~min}$ of the experiment according to the formula $\left(\mathrm{Fl}_{10 \min }-\mathrm{Fl}_{0 \text { min }}\right) /$ $\mathrm{FI}_{0 \text { min. }}$. The same formula was used to determine the relative fluorescence change in the period just prior to GVBD $(n=12) . * P<0.05$ at least, compared with rate of import in the presence of leptomycin $B$. that nuclear accumulation involves factors other than inhibition of export, most likely an increase in import.

\section{Discussion}

In this study we have used a cyclin B1-GFP fusion protein to investigate the role and dynamics of cyclin B1 during $\mathrm{MI}$ in living mouse oocytes. We have found that exogenous cyclin B1 can accelerate GVBD and override cAMPmediated arrest and had a concentration-dependent effect on polar body extrusion. The events of meiotic maturation were associated with changes in the localisation of cyclin B1-GFP. In GV stage oocytes, cyclin B1-GFP was retained in the cytoplasm by active nuclear export then, just prior to GVBD, cyclin B1-GFP translocated to the nucleus where it became associated with chromatin.

\section{Exogenous cyclin stimulates progression through meiosis}

The ability of cyclin B1-GFP to accelerate the rate of GVBD has demonstrated that GVBD in mouse oocytes is sensitive to the abundance of cyclin. The accelerated rate of GVBD was accompanied by an increase in MPF activity, some fivefold greater than the level of MPF that accompanied GVBD in control oocytes. These high levels of MPF are likely to explain the ability of exogenous cyclin B1 to override cAMP-mediated arrest (Ledan et al. 2001, present study). The sensitivity of GVBD to cyclin B1 
abundance has been reported in other species including Xenopus (Pines \& Hunt 1987, Westendorf et al. 1989, Li et al. 1997) and bovine (Levesque \& Sirard 1996, Robert et al. 2002) but, in these species, it was consistent with the need for new cyclin B synthesis to induce GVBD. In contrast, mouse oocytes do not require new protein synthesis to undergo GVBD (Clarke \& Masui 1983) and have been reported to have a sevenfold abundance of cyclin B1 over cdk1 (Kanatsu-Shinohara et al. 2000). Nevertheless, despite the apparent abundance of cyclin B1 in fully grown GV stage oocytes, our data show that exogenous cyclin B1-GFP is a potent activator of MPF activity.

How does excess exogenous cyclin B1 lead to the activation of MPF? One possibility is that, despite the reported excess of cyclin B, much of it may be sequestered (Westendorf et al. 1989, de Vantéry et al. 1997, Beckhelling et al. 2003, Terasaki et al. 2003). A small increase in 'free' exogenous cyclin B1 may provide a sufficient increase in pre-MPF available for cdc25 to initiate the auto-catalytic reaction leading to complete MPF activation (Hoffmann et al. 1993, Lincoln et al. 2002). This is consistent with the idea that activation of cdk1 behaves as a bi-stable switch, thereby ensuring oscillations between an interphase and M-phase state (Pomerening et al. 2003). This cyclin abundance-stimulated switch in activity provides a reasonable explanation for the early onset of MPF activity seen in our studies. The switch-like activation of proteins that regulate the cell cycle is also seen in the activation of MAP kinase (Ferrell \& Machleder 1998). This is consistent with our findings showing that MAP kinase was activated to the same extent irrespective of the level of MPF activity.

The fivefold increase in MPF activity over that seen in uninjected oocytes suggests that injected cyclin B1-GFP is contributing to the high levels of cdk1 activity seen. The extent of cdk1 activity may be regulated in part by the lack of a negative feedback normally provided by cdk1-dependent activation of the anaphase promoting complex (APC) (Pomerening et al. 2003). In Ml, APC activity is suppressed for $4-5 \mathrm{~h}$ after the initial activation of cdk 1 , probably via the activation of the mos/MAP kinase pathway and the spindle assembly checkpoint (Brunet et al. 2003, Tunquist \& Maller 2003, Wassmann et al. 2003). As such, in the absence of the inactivation pathway, the positive feedback pathways that lead to MPF activation may progress to completion with the extent of activation being related to the amount of cyclin B1 available for driving cdk1 activity.

\section{Cyclin B1 availability controls the duration of MI}

Cyclin injection also had effects on the timing of the first meiotic division. The ability of low doses of cyclin (10 and $20 \mathrm{pg}$ ) to accelerate the timing of extrusion of the first polar body is similar to previous studies where cyclin B was increased using cyclin B1 mRNA (Polanski et al. 1998, Ledan et al. 2001). The present data are in agreement with the finding that progression through $\mathrm{MI}$ is dependent on translation of cyclin B (Hampl \& Eppig 1995, Polanski et al. 1998). In addition to the acceleration of polar body extrusion at low doses, we found a dosedependent inhibition of polar body extrusion. The saturation of mechanisms for cyclin destruction during $\mathrm{MI}$ (Kobayashi et al. 1991) is the most likely explanation of this inhibitory effect of excess cyclin B1. This is supported by recent observations in mouse oocytes over-expressing cyclin B1-GFP showing that where destruction is delayed or insufficient, polar body extrusion does not take place (Ledan et al. 2001). In Xenopus oocytes cyclin destruction is not necessary for the transition from MI to MII (Peter et al. 2001, Taieb et al. 2001). In the mouse, the effects of excess cyclin B1 and more recent studies using nondestructible cyclin B1-GFP (Herbert et al. 2003) suggest that cyclin destruction is an important requirement in the progression from MI to MII.

\section{Cyclin B1 undergoes nuclear translocation prior to $G V B D$}

In GV stage mouse oocytes, cyclin B1-GFP was localised to the cytoplasm and excluded from the GV. In most oocytes, there were two accumulations of cyclin B1-GFP in the close proximity of the GV. These are likely to represent the oocyte centrosomes, also referred to as the microtubule organising centres since mouse oocytes do not contain centrioles. In somatic cells, Xenopus eggs and starfish oocytes, the centrioles are considered to be the site at which cdk1-cyclin B1 is first activated (Beckhelling et al. 2003, Jackman et al. 2003, Terasaki et al. 2003). Additional aggregates of cyclin B have been reported throughout the cytoplasm in Xenopus, clam and starfish oocytes (Westendorf et al. 1989, Beckhelling et al. 2003, Terasaki et al. 2003). These aggregates are detected by immunofluorescence and in starfish oocytes have also been detected using starfish or human cyclin B1-GFP fusion proteins (Terasaki et al. 2003). Using the same human fusion protein we have not observed any such aggregates in GV stage mouse oocytes. It will be interesting to determine whether the lack of any aggregates in mouse oocytes has any functional effects on the kinetics of MPF activation, as would be suggested by recent modelling studies (Slepchenko \& Terasaki 2003).

Cyclin B1-GFP was retained in the cytoplasm of GV stage oocytes through an active nuclear-export mechanism, consistent with findings in other systems (Hagting et al. 1989, 1999, Yang et al. 1998). Cyclin B1-GFP injected into the GV was exported while a similar size dextran was retained. In addition, the nuclear export inhibitor, leptomycin B, accelerated accumulation of cyclin B1-GFP in the $\mathrm{GV}$. These experiments demonstrated that cyclin B1 cycles between the GV and the cytoplasm and that cyclin B1 is retained in the cytoplasm of GV stage oocytes by a rate of export that outpaces the rate of import.

In the $10-15 \mathrm{~min}$ preceding GVBD, the balance was shifted such that import became dominant with the result 
that cyclin B1-GFP accumulates in the GV. This accumulation is due to active transport rather than passive diffusion through leaky nuclear pores (Lenart et al. 2003) since it accumulates to levels that exceed those in the cytoplasm. This is consistent with recent elegant experiments in starfish oocytes showing that the accumulation of cyclin B1-GFP precedes an increase in nuclear pore permeability by imaging the distribution of a $70 \mathrm{kDa}$ dextran simultaneously with cyclin B1-GFP (Terasaki et al. 2003). The rapid accumulation of cyclin B1-GFP just prior to GVBD suggests that, in addition to inhibition of nuclear export, an increase in import is necessary for driving cyclin B1-GFP translocation to the GV.

The tight temporal relationship between cyclin B1-GFP accumulation in the GV and GVBD suggests that nuclear accumulation of cyclin B1 is required for the rapid onset of GVBD. This is further supported by the observation that, in oocytes that remained arrested in the presence of dbcAMP, the cyclin B1-GFP remained cytoplasmic. In Xenopus oocytes, there is direct evidence that nuclear accumulation of cyclin B is necessary for GVBD since exogenous cyclin $B$ is sufficient to drive GVBD in the absence of progesterone but a phosphorylation mutant (cyclin $\mathrm{B} 1^{\text {ala }}$ ) that does not undergo nuclear translocation ( $\mathrm{Li}$ et al. 1997) is not. The addition of an nuclear localizing sequence to cyclin $\mathrm{B}^{\text {ala }}$ restores nuclear accumulation and the capacity to stimulate GVBD (Li et al. 1997). The fact that nuclear accumulation of cyclin B1-GFP precedes GVBD or NEBD is consistent with the role of cdk1-cyclin B1 in phosphorylation of nuclear lamins, an important step in initiation of nuclear envelope disassembly (Peter et al. 1990).

These studies highlight the importance of dynamic changes in the localization of cyclin B1 in the control of GVBD and MI. Since requirements for cyclin B synthesis have been met in GV stage oocytes, localisation may be one of the major mechanisms governing the timing of GVBD. In future studies it will be important to understand how the hormonal changes that induce GVBD in vivo link up with the mechanisms that stimulate the activation and re-localisation of cyclin B1-cdk1.

\section{Acknowledgements}

We thank Dr Jonathon Pines and his colleagues for generously supplying us with the purified cyclin B1-GFP fusion protein. Thanks are also due to Dr M Yoshida for sending us the leptomycin B and Geraint Thomas for assistance with setting up the kinase assays. We thank Jonathon Pines and Mark Larman for helpful discussions about the manuscript. This work was supported by an MRC Career Establishment Grant to J C.

\section{References}

Beckhelling C, Chang P, Chevalier S, Ford C \& Houliston E 2003 Pre$M$ phase-promoting factor associates with annulate lamellae in Xenopus oocytes and egg extracts. Molecular Biology of the Cell 14 1125-1137.

Brunet S, Pahlavan G, Taylor S \& Maro B 2003 Functionality of the spindle checkpoint during the first meiotic division of mammalian oocytes. Reproduction $126443-450$.
Casas E, Betancourt M, Bonilla E, Duculomb Y, Zayas H \& Trejo R 1999 Changes in cyclin B localisation during pig oocyte in vitro maturation. Zygote 7 21-26.

Chesnel F \& Eppig JJ 1995 Synthesis and accumulation of p34cdc2 and cyclin B in mouse oocytes during acquisition of competence to resume meiosis. Molecular Reproduction and Development $\mathbf{4 0}$ 503-508.

Choi T, Aoki F, Mori M, Yamashita M, Nagahama Y \& Kohmoto K 1991 Activation of p34cdc2 protein kinase activity in meiotic and mitotic cell cycles in mouse oocytes and embryos. Development $113789-795$.

Clarke HJ \& Masui Y 1983 The induction of reversible and irreversible chromosome decondensation by protein synthesis inhibition during meiotic maturation of mouse oocytes. Developmental Biology 97 291-301.

Clute P \& Pines J 1999 Temporal and spatial control of cyclin B1 destruction in metaphase. Nature Cell Biology 1 82-87.

Downs SM 1990 Protein synthesis inhibitors prevent both spontaneous and hormone-dependent maturation of isolated mouse oocytes. Molecular Reproduction and Development 27 $235-243$.

Draetta G, Luca F, Westendorf J, Brizuela L, Ruderman J \& Beach D 1989 Cdc2 protein kinase is complexed with both cyclin A and cyclin B: evidence for proteolytic inactivation of MPF. Cell $\mathbf{5 6}$ 829-838.

Evans T, Rosenthal ET, Youngblom J, Distel D \& Hunt T 1983 Cyclin: a protein specified by maternal mRNA in sea urchin eggs that is destroyed at each cleavage division. Cell 33 389-396.

Ferrell JE Jr \& Machleder EM 1998 The biochemical basis of an allor-none cell fate switch in Xenopus oocytes. Science $\mathbf{2 8 0}$ 895-898.

Fornerod M, Ohno M, Yoshida M \& Mattaj IW 1997 CRM1 is an export receptor for leucine-rich nuclear export signals. Cell $\mathbf{9 0}$ 1051-1060.

Fulton BP \& Whittingham DG 1978 Activation of mammalian oocytes by intracellular injection of calcium. Nature 273 149-151.

Gautier J, Minshull J, Lohka M, Glotzer M, Hunt T \& Maller JL 1990 Cyclin is a component of maturation-promoting factor from Xenopus. Cell $60487-494$.

Glotzer M, Murray AW \& Kirschner MW 1991 Cyclin is degraded by the ubiquitin pathway. Nature 349 132-138.

Hagting A, Karlsson C, Clute P, Jackman M \& Pines J 1998 MPF localisation is controlled by nuclear export. The EMBO Journal 17 4127-4138.

Hagting A, Jackman M, Simpson K \& Pines J 1999 Translocation of cyclin B1 to the nucleus at prophase requires a phosphorylationdependent nuclear import signal. Current Biology 9 680-689.

Hampl A \& Eppig JJ 1995 Translational regulation of the gradual increase in histone $\mathrm{H} 1$ kinase activity in maturing mouse oocytes. Molecular Reproduction and Development 40 9-15.

Hashimoto N \& Kishimoto T 1988 Regulation of meiotic metaphase by a cytoplasmic maturation-promoting factor during mouse oocyte maturation. Developmental Biology 126 242-252.

Herbert $M$, Levasseur $M$, Homer $H$, Yallop $K$, Murdoch A \& McDougall A 2003 Homologue disjunction in mouse oocytes requires proteolysis of securin and cyclin B1. Nature Cell Biology 5 1023-1025.

Hoffmann I, Clarke PR, Marcote MJ, Karsenti E \& Draetta G 1993 Phosphorylation and activation of human cdc25-C by cdc2-cyclin $\mathrm{B}$ and its involvement in the self-amplification of MPF at mitosis. The EMBO Journal 12 53-63.

Hunt T 1989 Maturation promoting factor, cyclin and the control of M-phase. Current Opinion in Cell Biology 1 268-274.

Jackman M, Lindon C, Nigg EA \& Pines J 2003 Active cyclin B1Cdk1 first appears on centrosomes in prophase. Nature Cell Biology 5 143-148.

Kanatsu-Shinohara M, Schultz RM \& Kopf GS 2000 Acquisition of meiotic competence in mouse oocytes: absolute amounts of 
p34cdc2, cyclin B1, cdc25C, and wee1 in meiotically incompetent and competent oocytes. Biology of Reproduction 63 1610-1616.

Kobayashi H, Minshull J, Ford C, Golsteyn R, Poon R \& Hunt T 1991 On the synthesis and destruction of A- and B-type cyclins during oogenesis and meiotic maturation in Xenopus laevis. Journal of Cell Biology 114 755-765.

Kubiak JZ, Weber M, de Pennart H, Winston NJ \& Maro B 1993 The metaphase II arrest in mouse oocytes is controlled through microtubule-dependent destruction of cyclin B in the presence of CSF. The EMBO Journal 12 3773-3778.

Kudo N, Wolff B, Sekimoto T, Schreiner EP, Yoneda Y, Yanagida M, Horinouchi S \& Yoshida M 1998 Inhibition of signal-mediated nuclear export by direct binding to CRM1. Experimental Cell Research 242 540-547.

Labbé JC, Picard A, Peaucellier G, Cavadore JC, Nurse P \& Dorée M 1989 Purification of MPF from starfish: identification as the $\mathrm{H} 1$ histone kinase p34cdc2 and a possible mechanism for its periodic activation. Cell $57253-263$.

Ledan E, Polanski Z, Terret M-E \& Maro B 2001 Meiotic maturation of the mouse oocyte requires an equilibrium between cyclin B synthesis and degradation. Developmental Biology 232 400-413.

Lenart P, Rabut G, Daigle N, Hand AR, Terasaki M \& Ellenberg J 2003 Nuclear envelope breakdown in starfish oocytes proceeds by partial NPC disassembly followed by a rapidly spreading fenestration of nuclear membranes. Journal of Cell Biology $\mathbf{1 6 0}$ 1055-1068.

Levesque JT \& Sirard MA 1996 Resumption of meiosis is initiated by the accumulation of cyclin B in bovine oocytes. Biology of Reproduction 55 1427-1436.

Li J, Meyer AN \& Donoghue DJ 1997 Nuclear localization of cyclin B1 mediates its biological activity and is regulated by phosphorylation. PNAS 94 502-507.

Lincoln AJ, Wickramasinghe D, Stein P, Schultz RM, Palko ME, De Miguel MP, Tessarollo L \& Donovan PJ 2002 Cdc25b phosphatase is required for resumption of meiosis during oocyte maturation. Nature Genetics 30 446-449.

Mitra J \& Schultz RM 1996 Regulation of the acquisition of meiotic competence in the mouse: changes in the subcellular localization of cdc2, cyclin B1, cdcd25C and wee1, and in the concentration of these proteins and their transcripts. Journal of Cell Science $\mathbf{1 0 9}$ 2407-2415.

Moos J, Visconti PE, Moore GD, Schultz RM \& Kopf GS 1995 Potential role of mitogen-activated protein kinase in pronuclear envelope assembly and disassembly following fertilization of mouse eggs. Biology of Reproduction 53 692-699.

Murray AW \& Kirschner MW 1989 Cyclin synthesis drives the early embryonic cell cycle. Nature 339 275-280.

Ookata K, Hisanaga S, Okano T, Tachibana K \& Kishimoto T 1992 Relocation and distinct subcellular localization of p34cdc2-cyclin $\mathrm{B}$ complex at meiosis reinitiation in starfish oocytes. The $E M B O$ Journal 11 1763-1772.

Peter M, Nakagawa J, Dorée M, Labbé JC \& Nigg EA 1990 In vitro disassembly of the nuclear lamina and M-phase specific phosphorylation of lamins by cdc2 kinase. Cell 61 591-602.

Peter M, Castro A, Lorca T, Le Peuch C, Magnaghi-Jaulin L, Doree M \& Labbe JC 2001 The APC is dispensable for first meiotic anaphase in Xenopus oocytes. Nature Cell Biology 3 83-87.

Pines J 1999 Four-dimensional control of the cell cycle. Nature Cell Biology 1 E73-E79.

Pines J \& Hunt T 1987 Molecular cloning and characterization of the mRNA for cyclin from sea urchin eggs. The EMBO Journal 6 2987-2995.

Pines J \& Hunter T 1991 Human cyclins A and B are differentially located in the cell and undergo cell cycle dependent nuclear transport. Journal of Cell Biology 115 1-17.
Pines J \& Hunter T 1994 The differential localization of human cyclins $\mathrm{A}$ and $\mathrm{B}$ is due to a cytoplasmic retention signal in cyclin B. The EMBO Journal $133772-3781$.

Polanski Z, Ledan E, Brunet S, Louvet S, Kubiak JZ, Verlhac M-H \& Maro B 1998 Cyclin synthesis controls the progression of meiotic maturation in mouse oocytes. Development 125 4989-4997.

Pomerening JR, Sontag ED \& Ferrell JE Jr 2003 Building a cell cycle oscillator: hysteresis and bistability in the activation of $\mathrm{Cdc} 2$. Nature Cell Biology 5 346-351.

Robert C, Hue I, McGraw S, Gagne D \& Sirard MA 2002 Quantification of cyclin B1 and p34(cdc2) in bovine cumulus-oocyte complexes and expression mapping of genes involved in the cell cycle by complementary DNA macroarrays. Biology of Reproduction $\mathbf{6 7}$ 1456-1464.

Slepchenko BM \& Terasaki M 2003 Cyclin aggregation and robustness of bio-switching. Molecular Biology of the Cell $\mathbf{1 4}$ 4695-4706.

Taieb FE, Gross SD, Lewellyn AL \& Maller JL 2001 Activation of the anaphase-promoting complex and degradation of cyclin B is not required for progression from Meiosis I to II in Xenopus oocytes. Current Biology 11 508-513.

Takizawa CG \& Morgan DO 2000 Control of mitosis by changes in the subcellular location of cyclin-B1-Cdk1 and Cdc25C. Current Opinion in Cell Biology 12 658-665.

Tay J, Hodgman R \& Richter JD 2000 The control of cyclin B1 mRNA translation during mouse oocyte maturation. Developmental Biology 221 1-9.

Terasaki M, Okumura E, Hinkle B \& Kishimoto T 2003 Localization and dynamics of $\mathrm{Cdc} 2$-cyclin B during meiotic reinitiation in starfish oocytes. Molecular Biology of the Cell 14 4685-4694.

Toyoshima F, Moriguchi T, Wada A, Fukuda M \& Nishida E 1998 Nuclear export of cyclin B1 and its possible role in the DNA damage-induced G2 checkpoint. The EMBO Journal 17 2728-2735.

Tunquist BJ \& Maller JL 2003 Under arrest: cytostatic factor (CSF)mediated metaphase arrest in vertebrate eggs. Genes and Development 17 683-710.

de Vantéry C, Stutz A, Vassalli JD \& Schorderet-Slatkine S 1997 Acquisition of meiotic competence in growing mouse oocytes is controlled at both translational and posttranslational levels. Developmental Biology 187 43-54.

Verlhac MH, de Pennart H, Maro B, Cobb MH \& Clarke HJ 1993 MAP kinase becomes stably activated at metaphase and is associated with microtubule-organizing centers during meiotic maturation of mouse oocytes. Developmental Biology 158 330-340.

Wassmann K, Niault T \& Maro B 2003 Metaphase I arrest upon activation of the Mad2-dependent spindle checkpoint in mouse oocytes. Current Biology 13 1596-1608.

Westendorf JM, Swenson KI \& Ruderman JV 1989 The role of cyclin B in meiosis I. Journal of Cell Biology 108 1431-1444.

Winston N 1997 Stability of cyclin B protein during meiotic maturation and the first meiotic cell cycle division in mouse oocyte. Biology of the Cell 89 211-219.

Yang J, Bardes ES, Moore JD, Brennan J, Powers MA \& Kornbluth S 1998 Control of cyclin B1 localization through regulated binding of the nuclear export factor CRM1. Genes and Development 12 2131-2143.

Yang J, Song H, Walsh S, Bardes ESG \& Kornbluth S 2001 Combinatorial control of Cyclin B1 nuclear trafficking through phosphorylation at multiple sites. Journal of Biological Chemistry 276 3604-3609.

Received 7 February 2004

First decision 19 March 2004

Accepted 22 April 2004 